\title{
Punicalagin isolated from Punica granatum husk can decrease the inflammatory response in RAW 264.7 macrophages
}

\author{
Mehmet Berköz $^{1^{*}}$ and Oruc Allahverdiyev ${ }^{2}$ \\ ${ }^{1}$ Department of Pharmaceutical Biotechnology, Faculty of Pharmacy, Yuzuncu Yil University, Van, Turkey \\ ${ }^{2}$ Department of Pharmacology, Faculty of Pharmacy, Yuzuncu Yil University, Van, Turkey
}

\begin{abstract}
Punicalagin, a natural occuring phytochemical extracted from pomegranate (Punica granatum) husk shows antioxidant and anti-tumoral activities. Previous studies have shown that Punica granatum can scavenge reactive oxygen intermediates (ROIs) and suppress the biosynthesis of prostaglandins (PGs). However, the anti-inflammatory property of punicalagin has not yet been elucidated. For this aim, we purposed to display the anti-inflammatory effects of punicalagin via measuring prostaglandin $\mathrm{E}_{2}\left(\mathrm{PGE}_{2}\right)$ and nitric oxide $(\mathrm{NO})$ production and cyclooxygenase-2 (COX-2) and inducible nitric oxide synthase (iNOS) gene expression in LPS induced RAW 264.7 macrophages. LPS-stimulated RAW 264.7 macrophages were treated with punicalagin at concentrations of $0-10 \mu \mathrm{M}$. NO levels in all groups were measured by the Griess method and $\mathrm{PGE}_{2}$ levels by ELISA kit. COX-2 and $i$ NOS gene expression levels were measured using Real Time PCR. According to our experiments, punicalagin decreased the production of $\mathrm{PGE}_{2}$ and $\mathrm{NO}$ by RAW 264.7 macrophages in a dose-dependent manner without affecting the viability of cells. Punicalagin attenuated the mRNA expression of $i N O S$ and COX-2 of murine macrophages in a concentration dependent manner. Thus, the inhibition of $\mathrm{NO}$ and $\mathrm{PGE}_{2}$ production is at least partly because of the suppression of the transcription of the iNOS and COX-2 gene, respectively. In conclusion, punicalagin is a potent natural compound in inhibiting the inflammatory mediators. Its action can be delivered in vivo through an appropriate feeding scheme. Because the lower toxicity of punicalagin, it might be a suitable compound that can be used for clinical applications.
\end{abstract}

Key Words: Punicalagin, inflammation, RAW 264.7 macrophages, nitric oxide, prostaglandin $\mathrm{E}_{2}$

\section{Introduction}

Inflammation is an important immune response to defend the body from invasion of tissue injury, chemical irritation or microbial pathogen infection. It has a vital function in the immune response to combat foreign invaders of the body $(1,2)$. Under inflammatory condition, mast cells secrete chemokines and pro-inflammatory cytokines that cause angiectasis, increase permeability, and subsequently recruit leukocytes, macrophages and a wide range of immune cells to invade the affected area. Leukocytes and macrophages infiltrate the damaged regions, remove the irritation and repair the tissue. RAW 264.7 murine macrophages obtained from the tissue of Abelson murine leukemia virus-induced tumor in male adult BALB/c Mus musculus is one of the common cell line model in inflammatory studies since its lipopolysaccharide (LPS) induced state exhibits these typical functional and morphological phenotypes $(3,4)$. LPS triggers the secretion of pro-inflammatory mediators such as prostaglandin $\mathrm{E}_{2}\left(\mathrm{PGE}_{2}\right)$ and nitric oxide $(\mathrm{NO})$ which are created by the inducible nitric oxide synthase (iNOS) and cyclooxygenase-2 (COX-2), respectively. Inhibition of these pro-inflammatory mediators is a critical goal to protect the organism against the inflammation. Thus, many compounds believed to have anti-inflammatory activity have been applied to RAW 264.7 macrophages for treating the inflammatory response $(5,6)$.

Pomegranate (Punica granatum L., Punicaceae) has been widely used in ayuverdic medicine and other traditional medicine. Pomegranate leaf extract exhibits antihelmintic, antiparasitic, antidiarrhoeal, antioxidant, antitumoral and anti-inflammatory properties (7). Two types of polyphenols are found in pomegranate; anthocyanins (delphinidin, cyaniding and pelargonidin), responsible for the red color of the fruit, and hydrolysable tannins, mainly ellagitannins. These polyphenols are responsible for $90 \%$ of the antioxidant capacity of pomegranate and punicalagin alone undertakes more than $50 \%$ of this antioxidant property (8). Punicalagin is a hydrolyzable tannin and it is 
considered to be the main compound in pomegranate husk (9). Punicalagin is reported to have anti-cancer, antiatherosclerotic and antiobesity properties (10). Although many researches have reported the anti-inflammatory feature of pomegranate leaf extract, effects of punicalagin obtained from pomegranate leaf extract is not known clearly $(11,12)$.

Better discerning of the anti-inflammatory effects of punicalagin may lead to further development of new strategies for dysregulated inflammatory prevention at many sites. Therefore, in this study, we purposed to investigate the anti-inflammatory effects of punicalagin via measuring $\mathrm{PGE}_{2}$ and $\mathrm{NO}$ production and COX-2 and iNOS gene expression in LPS induced macrophages.

\section{Materials and methods}

\section{Cell culture}

RAW 264.7 macrophages were purchased from American Type Culture Collection (ATCC) and the cells were incubated at $37^{\circ} \mathrm{C}$ and $5 \% \mathrm{CO}_{2}$. All the chemicals used in our study were purchased from Sigma-Aldrich and Merck companies and all products were in cell culture purity. The cells were passaged when they were cultivated at a density of $70 \sim 80 \%$ full of the dish. Medium was removed and $1 \times$ Phosphate buffered saline (PBS) was used to wash the dish. We subsequently tapped the dish and used fresh medium to wash down the cells. The fallen cells were collected in centrifuge tube, and we centrifuged the tube with $1000 \mathrm{rpm}$ for 5 minute. After that, the supernatant was sucked up and the pellet was dispersed by fresh medium. Finally, the cells were seeded in dishes or wells with a proper density.

After the cells grew stably and reached at a density of $2-3 \times 10^{6}$ cells $/ \mathrm{mL}$, the medium was removed and $1 \times$ PBS was used to gently wash the dish. The medium was replaced serum-free DMEM. The cells were induced by incubation in medium containing $0.1 \mu \mathrm{g} / \mathrm{mL}$ LPS (E. coli 0127: E8). Punicalagin was dissolved in DMEM. These dissolution compounds were treated cells together with LPS.

\section{Cell viability assay}

The cellular toxicity of punicalagin was determined by (3-(4,5-Dimethylthiazol-2-yl)-2,5Diphenyltetrazolium Bromide (MTT) assay (13). After $24 \mathrm{hr}$ of incubation with the cells, punicalagin were removed by washing the cultured cells three times with medium. Then, the cells were collected by centrifugation and the pellets were suspended in fresh medium. The MTT solution was prepared using phosphate buffered solution (PBS) at $5 \mathrm{mg} / \mathrm{mL}$ concentration. MTT solution was added to the culture plate with cells and then incubated at $37^{\circ} \mathrm{C}$ for 4 hours. The solution of $0.4 \mathrm{~N} \mathrm{HCl}$ in isopropyl alcohol was added to the culture well to dissolve the formed tetrazolium salt. The results were measured by ELISA which read the absorption at $540 \mathrm{~nm}$ in ELISA microplate reader (Thermo Fisher Scientific, Wathham, MA, USA). The experiment was repeated by three times. Medium without cells was used as blank.

\section{Determination of NO production}

Nitrite and peroxynitrite levels in the cell supernatant were measured using the Griess method (14) to determine NO level in the cell culture medium. Nitrite and peroxynitrite are stable end products of $\mathrm{NO}$ in in vitro system. By this way, the level of NO synthesis by iNOS can be evaluated. Griess reagent was prepared by mixing 1 volume of $1 \%$ sulfanilamide solution and 1 volume of $0.1 \% \mathrm{~N}$-l-naphthyl ethlenediamine dihydrochloride prepared in $2.5 \% \mathrm{H}_{3} \mathrm{PO}_{4}$. Tested cells were treated by $0.1 \mu \mathrm{g} / \mathrm{mL}$ LPS. The tested compounds were added in gradient concentrations into the medium with cultured cells before the adding of the stimulants. After $24 \mathrm{hr}, 48 \mathrm{hr}$ and 72 $\mathrm{hr}$ of stimulation, $50 \mu \mathrm{L}$ of supernatant of the culture wells was taken and mixed with $50 \mu \mathrm{L}$ freshly prepared Griess reagent. After incubation at room temperature for 10 minutes, the ELISA plate was centrifuged for 5 minutes at $1000 \mathrm{rpm}$. The results were obtained from ELISA by reading the absorption at $540 \mathrm{~nm}$. This reaction was repeated three times for each sample. Accumulation of nitrite was calculated as nmol of nitrite per $10^{6}$ cells from three independent experiments. The culture medium without cells was used as blank. Sodium nitrate $\left(\mathrm{NaNO}_{2}\right)$ was diluted to $3 \mu \mathrm{M}, 25 \mu \mathrm{M}, 50 \mu \mathrm{M}$ and $100 \mu \mathrm{M}$ and used as standard solutions.

\section{Determination of $\mathrm{PGE}_{2}$ production}

Prostaglandins (PGs) are lipid compounds derived from arachidonic acid. Like all PGs, $\mathrm{PGE}_{2}$ can be produced by several tissues including the gut, the uterus, blood vessels, bladder, placenta, brain and cells of the immune system. In some pathologic conditions such as tissue injury, inflammation and many cancers the production of $\mathrm{PGE}_{2}$ is increased in bodily fluids. The principle of $\mathrm{PGE}_{2}$ immunoassay is that $\mathrm{PGE}_{2}$ from culture medium competes with horseradish peroxidase (HRP)labeled PGE 2 . After both $\mathrm{PGE}_{2}$ and HRP-labeled $\mathrm{PGE}_{2}$ bind to the antibody sites, a substrate 
solution is added to evaluate the bound enzyme activity. The concentration of $\mathrm{PGE}_{2}$ present in the sample is inversely proportional to the intensity of the color at the end of the immunoassay.

\section{Gene expression analysis}

$\boldsymbol{R N A}$ extraction: Culture medium was removed after 4 or 5 hours of incubation. $300 \mu \mathrm{L}$ lysis buffer was added per $10^{6}$ cells. Then, $100 \mu \mathrm{L}$ of protein/DNA precipitating buffer was mixed and centrifuged to remove the protein and DNA. Supernatant was removed to new eppendorf tube and mixed with $300 \mu \mathrm{L}$ isopropanol. The tube was put in $-70{ }^{\circ} \mathrm{C}$ freezer for more than 24 hours to precipitate RNA. RNA pellet was washed by $70 \%$ ethanol and then dissolved in DEPC-water. For tissue samples, TriReagent (Sigma) was used to homogenize the tissue samples in homogenizers. Chloroform was added to remove DNA and protein. After centrifugation, the water phase was removed to a new tube and 1 volume isopropanol was mixed to precipitate RNA. RNA was washed by $70 \%$ ethanol to remove isopropanol and dissolved into DEPC-water. The concentration of extracted RNA was quantitated by measuring the absorption in $\mathrm{OD}_{260}$ and $\mathrm{OD}_{280}$ in spectrophotometer. The ratio of $\mathrm{OD}_{260} / \mathrm{OD}_{280}$ is between 1.6 to 1.8 for samples were used for the following experiments.

cDNA synthesis: Total RNA extracted from cultured cells and tissue samples were used as template for reverse transcription. $300 \mathrm{ng}$ total RNA was added to $25 \mu \mathrm{L}$ total volume of reaction mix containing 0.6 mM dNTPs (Promega), 10 units Rnase inhibitor (Clonetech), O1igo(dT) 15 primer (Promega) and 100 units of MMLV-RT (Promega). The whole reaction mix was incubated for 20 minutes at $42^{\circ} \mathrm{C}$. Then, the activity of reverse transcriptase was stopped via heating at $94^{\circ} \mathrm{C}$ for 5 minutes. The synthesized cDNA was chilled on ice immediately for PCR reaction or stored at $-70^{\circ} \mathrm{C}$ for later analysis.

Real time PCR Analysis: The production of PCR products was determined by measuring the SYBR Green fluorescence signal. SYBR Green DNA enters small, large cavities in double strands. The unbound dye in the solution emits very low fluorescence. However, as the binding of SYBR Green to DNA increases, the fluorescence emission at a wavelength of $530 \mathrm{~nm}$ rises. During PCR, the increase in SYBR Green fluorescence composition increases with the proportion of directly produced double-stranded DNA. Real Time PCR was performed in the direction of the manufacturer using the Invitrogen Universal EXPRESS SYBR Greener qPCR SuperMixes and Two-Step qRT-PCR kit on the Roche LightCycler 480 II device. The following PCR primers were used; $(\beta$-actin forward; 5'-AGG TCA TCA CTA TTG GCA AC-3', $\beta$-actin reverse; 5'-ACT CAT CGT ACT CCT GCT TG-3', COX-2 forward; 5'GTC TGA TGA TGT ATG CCA CAA TCT G3', COX-2 reverse; 5'-GAT GCC AGT GAT AGA GGG TGT TAA A-3', iNOS forward; 5'-CCC TTC CGA AGT TTC TGG CAG CAG C-3' and iNOS reverse, 5'-GGC TGT CAG AGC CTC GTG GCT TTG G-3' (15). In our study, $\beta$-actin gene was used as the house-keeping gene. Concentration values of iNOS and COX-2 gene expression of each sample were proportional to the concentration of $\beta$-actin of the same sample. The obtained values were multiplied by 100 and gene expression levels were calculated for each sample and the results obtained were compared. Relative quantitation was also used when expression changes were calculated and the expression of iNOS and COX-2 genes in LPS and different concentration of punicalagin treated RAW 264.7 macrophages was expressed as folds, assuming gene expression values 1 in no-treatment controls.

\section{Statistical analysis}

All experiments were repeated three times. The values were determined as mean \pm SE (standard error). SPSS (version 15.0 for windows) was used for determining the treatment effects by one-way ANOVA. The difference or correlation was considered to be statistically significant if $\mathrm{p}<0.05$ in the obtained results.

\section{Results}

\section{Cytotoxicity of punicalagin in RAW 264.7 macrophages}

Prior to evaluation of the $\mathrm{NO}$ and $\mathrm{PGE}_{2}$ inhibitory effect of punicalagin, we first determined its non-cytotoxic concentration in LPS-stimulated RAW 264.7 macrophages using MT'T assays. In this study we have applied to punicalagin RAW 264.7 macrophages at concentrations of $0,2.5,5,7.5,10,20,30,40,50$, $100,150,150,250$ and $500 \mu \mathrm{M}$. Concentrations of $0,2.5,5,7.5$ and $10 \mu \mathrm{M}$ of punicalagin were used in our experimental study, with a maximum of $10 \%$ cytotoxicity, in other words at least $90 \%$ cell viability (Figure 1). In our study, the LD50 concentration of punicalagin for the RAW 264.7 macrophages was found to be $895.01 \mu \mathrm{M}$. 
Effects of punicalagin on NO and $\mathrm{PGE}_{2}$ production in LPS-stimulated RAW 264.7 macrophages

We measured $\mathrm{NO}$ and $\mathrm{PGE}_{2}$ production in LPSstimulated RAW 264.7 macrophages for determining the possible anti-inflammatory effects of punicalagin. As shown in Figure 2, NO production and in Figure 3, $\mathrm{PGE}_{2}$ production was substantially higher in LPS-treated cells than in the untreated control cells $(\mathrm{p}<0.05)$. The addition of $2.5,5,7.5$ and $10 \mu \mathrm{M}$ punicalagin caused $31.74 \%, 38.02 \%, 43.35 \%$ and $50.18 \%$ reduction in LPS-induced NO production, respectively (Figure
2 and 3) and the addition of 2.5, 5, 7.5 and $10 \mu \mathrm{M}$ punicalagin caused $5.73 \%, 12.54 \%, 21.09 \%$ and $25.34 \%$ reduction in LPS-induced $\mathrm{PGE}_{2}$ production, respectively (Figure 3 ). The decrease in NO production was statistically significant for all punicalagin concentrations $(\mathrm{p}<0.05)$. The decrease in $\mathrm{PGE}_{2}$ production was not statistically significant in the groups treated with punicalagin at concentrations of 2.5 and $5 \mu \mathrm{M}(\mathrm{p}>0.05)$, but a statistically significant difference was observed in the groups treated with punicalagin at concentrations of 7.5 and $10 \mu \mathrm{M}(\mathrm{p}<0.05)$.

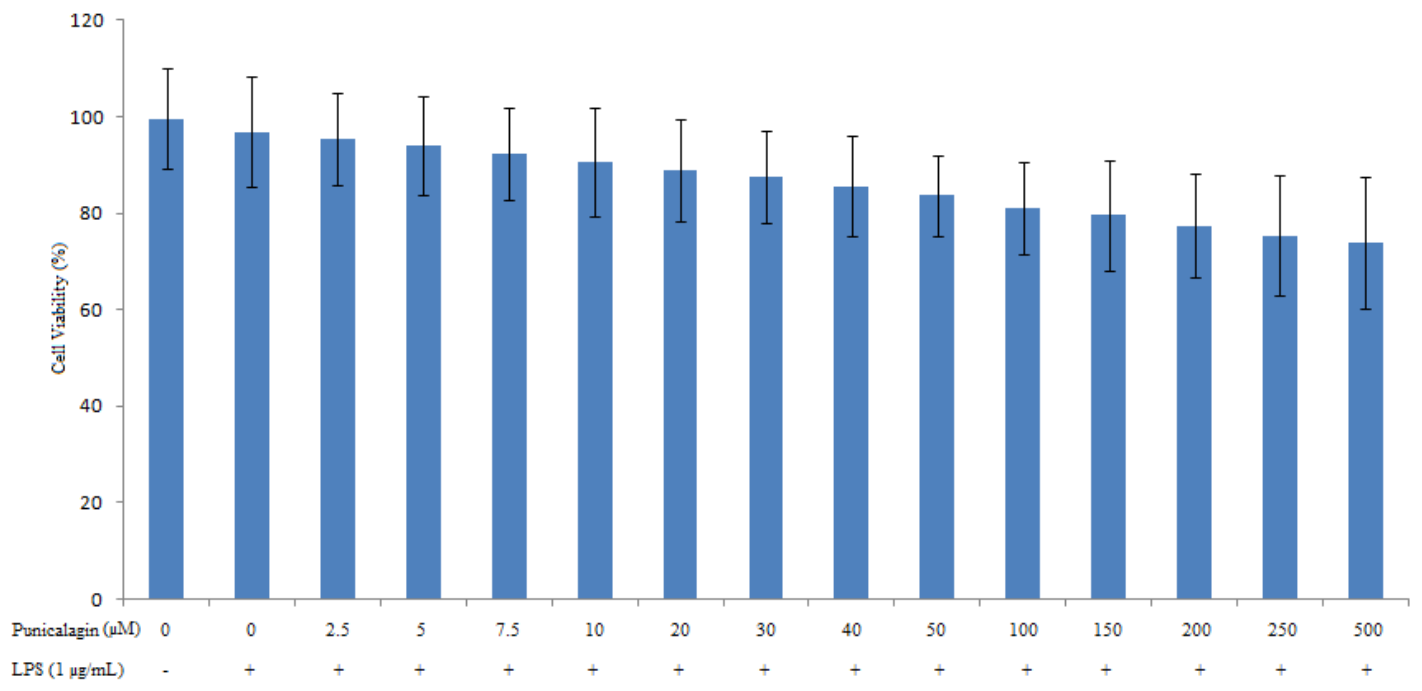

Fig. 1. Effect of punicalagin on the cell viability of RAW 264.7 macrophages.

There was no statistically differences between the groups ( $p>0.05)$.

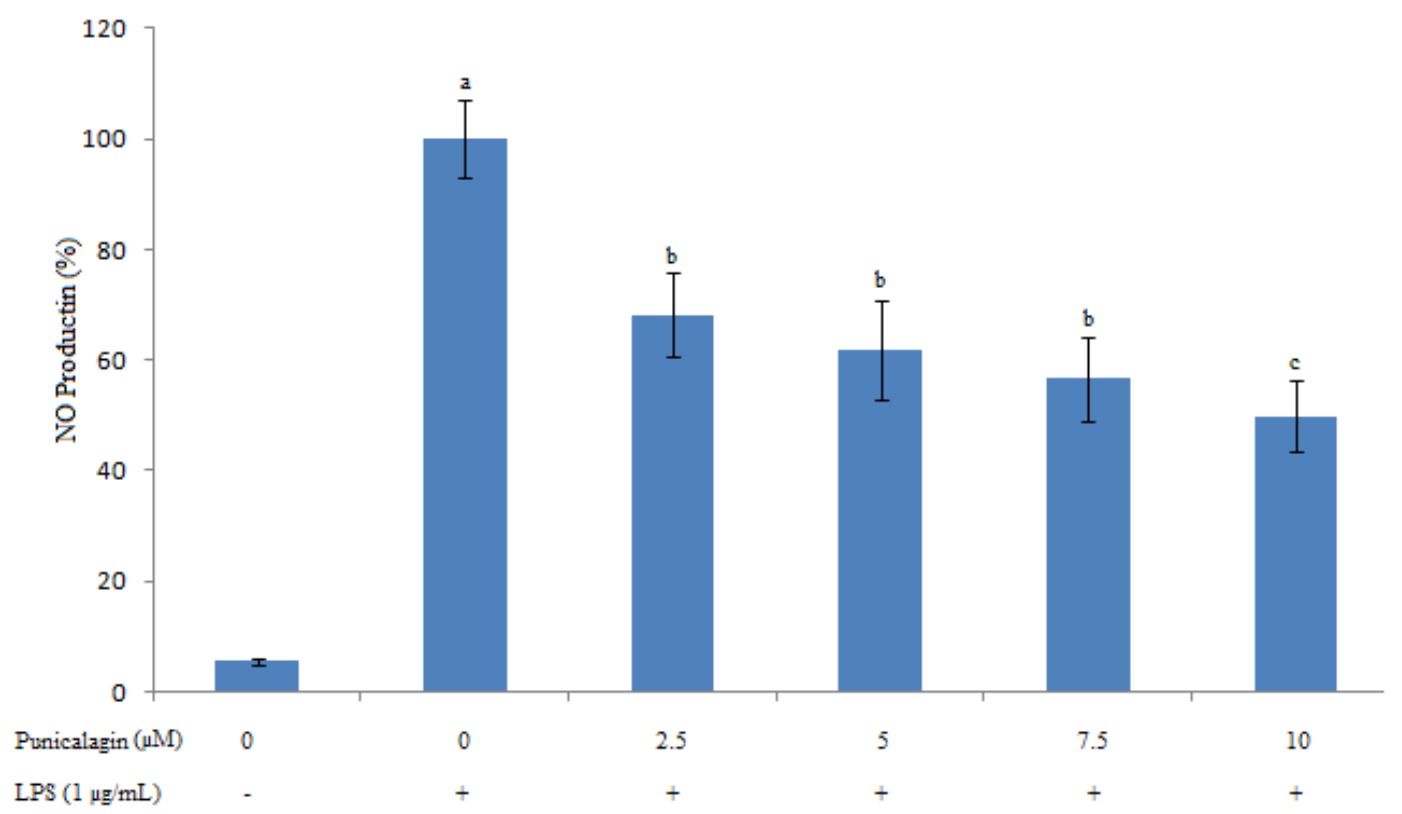

Fig. 1. Effect of punicalagin on the cell viability of RAW 264.7 macrophages.

There was no statistically differences between the groups ( $>0.05)$. 


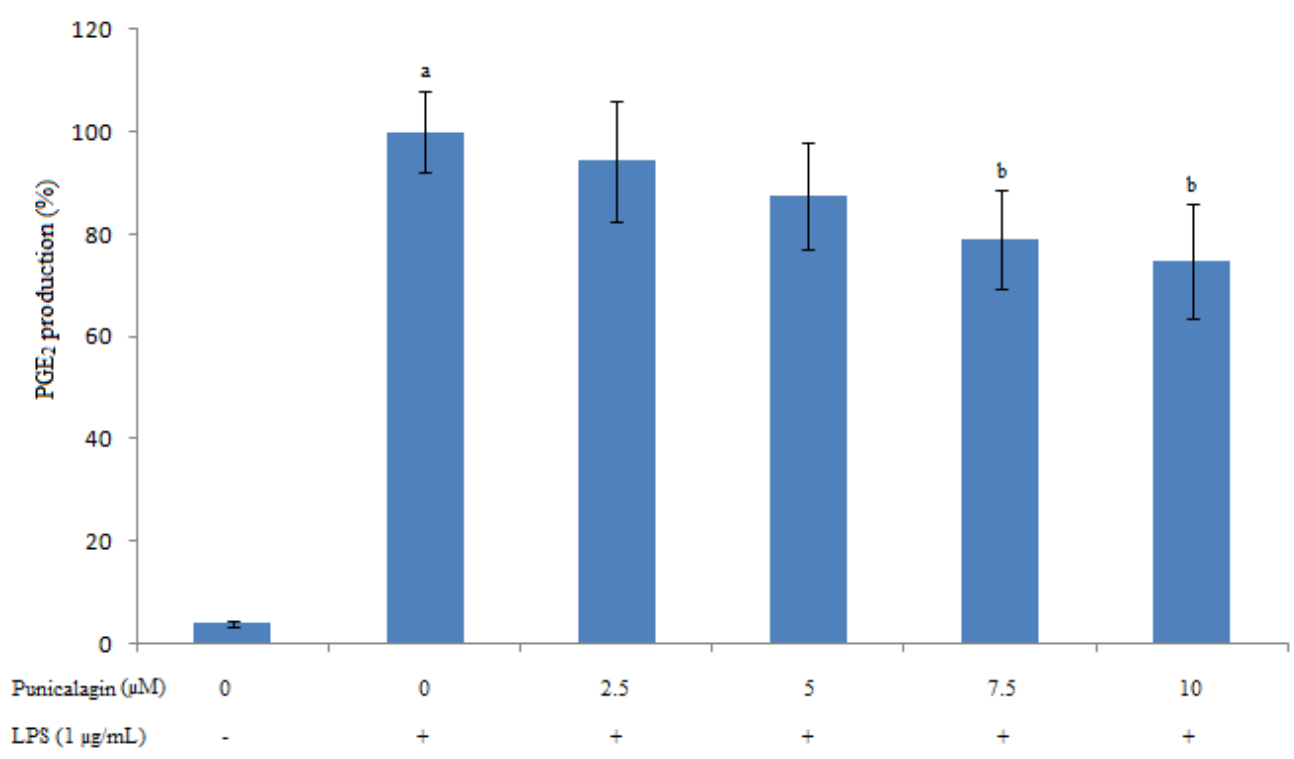

Fig. 3. Effects of punicalagin on LPS-induced PGE 2 production in RAW 264.7 macrophages. ${ }^{a} \mathrm{p}<0.05$ compared with the control cells, and ${ }^{\mathrm{b}} \mathrm{p}<0.05$ compared with the LPS-only treated cells.

Effect of punicalagin on iNOS and COX-2 mRNA expression in LPS-stimulated RAW 264.7 macrophages

RT-PCR was performed to determine whether the inhibitory effects of punicalagin on $\mathrm{NO}$ and $\mathrm{PGE}_{2}$ production were related to the mRNA expression of $i N O S$ and COX-2. As shown in Figure 4, iNOS mRNA expression level and in Figure 5, COX-2 mRNA expression level were substantially higher in LPS-treated cells than in the untreated control cells $(p<0.05)$. The addition of $2.5,5,7.5$ and 10 $\mu \mathrm{M}$ punicalagin caused $34.51 \%, 46.09 \%, 54.18 \%$ and $62.7 \%$ reduction in LPS-induced iNOS mRNA expression, respectively (Figure 4) and the addition of $2.5,5,7.5$ and $10 \mu \mathrm{M}$ punicalagin caused $32.01 \%, 40.84 \%, 48.65 \%$ and $55.68 \%$ reduction in LPS-induced COX-2 mRNA expression level, respectively (Figure 5). It has been demonstrated that all of the punicalagin concentrations performed resulted in a statistically significant decrease in both iNOS and COX-2 gene expression $(\mathrm{p}<0.05)$.

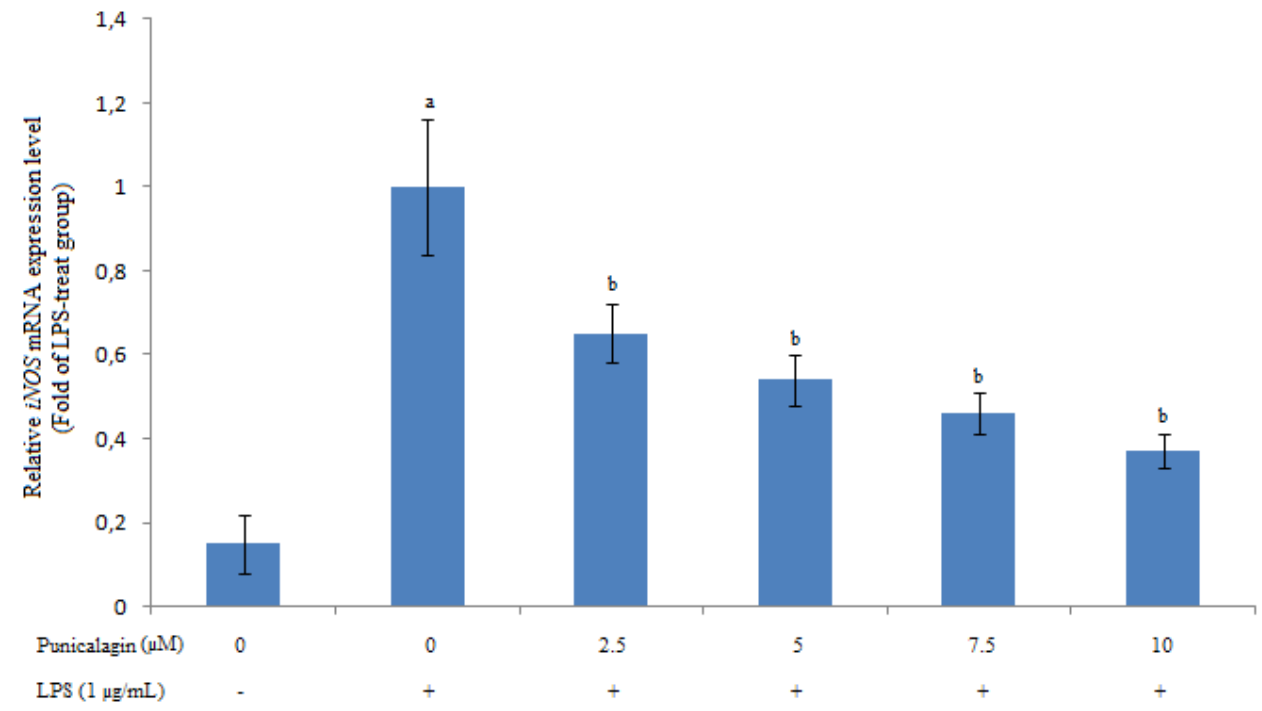

Fig. 4. Effects of punicalagin on LPS-induced iNOS gene expression in RAW 264.7 macrophages.

${ }^{\mathrm{a}} \mathrm{p}<0.05$ compared with the control cells, and ${ }^{\mathrm{b}} \mathrm{p}<0.05$ compared with the LPS-only treated cells.

East J Med Volume:22, Number:2, April-June/2017 


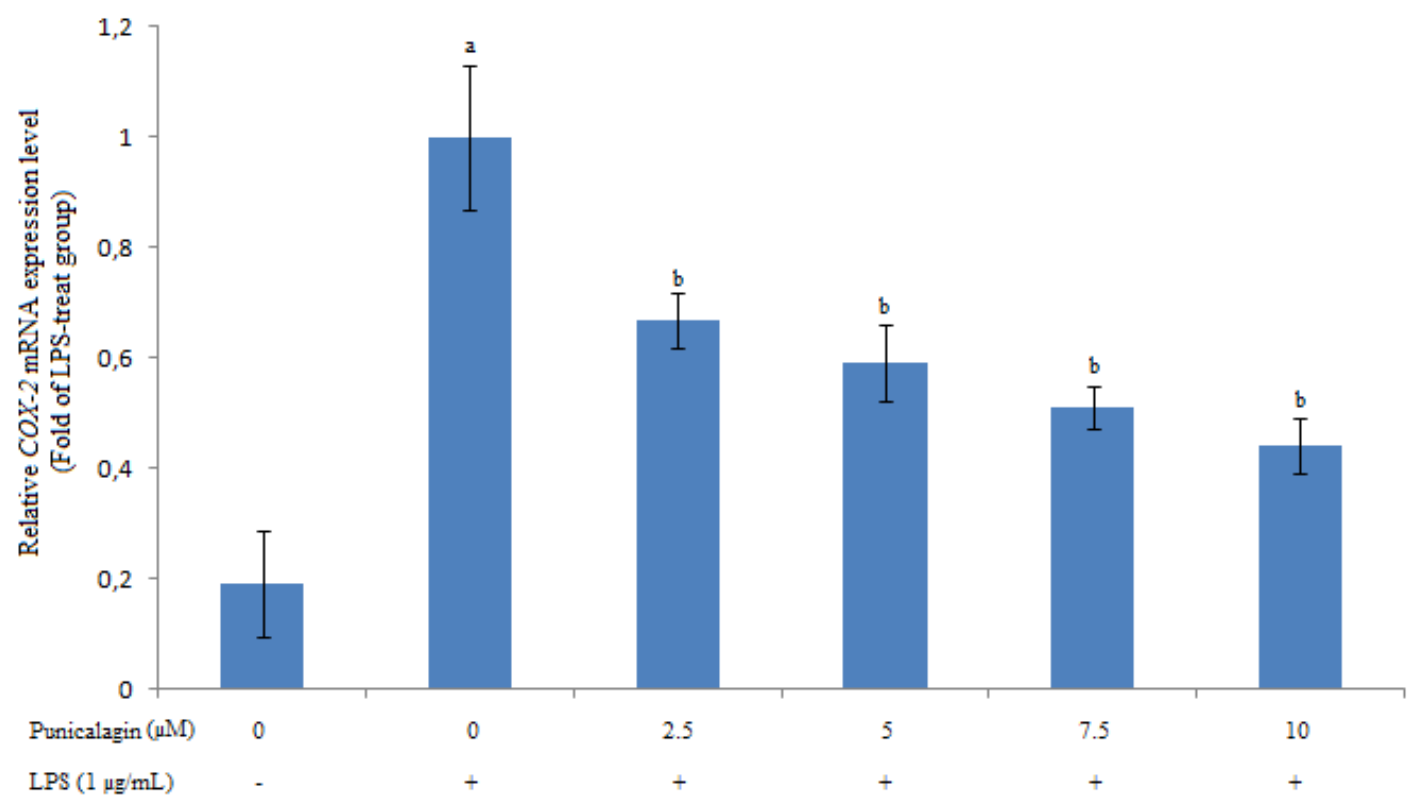

Fig. 5. Effects of punicalagin on LPS-induced COX-2 gene expression in RAW 264.7 macrophages.

${ }^{\mathrm{a}} \mathrm{p}<0.05$ compared with the control cells, and ${ }^{\mathrm{b}} \mathrm{p}<0.05$ compared with the LPS-only treated cells.

\section{Discussion}

Inflammation is a protective reaction orchestrated by the immune system to noxious stimuli and events, such as infection, tissue injury, and tissue malfunction. Its main purpose is to facilitate the removal of the stimuli, as well as the initiation of the healing process for the damaged cells or tissues. Inflammation is categorized as acute, localized chronic, and systemic chronic (16). The cellular and molecular mechanisms involved in acute inflammation, which are triggered commonly by infection and to some degree tissue injury, are well understood and characterized. However, much less is known about the causes and mechanisms of localized and systemic chronic inflammation, which are associated with a plethora of diseases - diabetes, obesity, insulin resistance, coronary heart disease, cardiovascular disease, cancer, Alzheimer's, asthma, inflammatory bowel disease, and arthritis (17).

Since murine macrophages, RAW 264.7 cell lines, are one of the common cell line models in inflammatory studies, we applied them as a model cell line in our study. They were isolated from the tissue of Abelson murine leukemia virus-induced tumor in male adult BALB/c mice. The culture property of the cells is adherent. In the research, a classical model of macrophage stimulation is bacterial endotoxin, lipopolysaccharide (LPS)
$(18,19)$. It is a classical and well-known model of macrophage stimulation. After LPS binds to the receptor, the signaling pathways are induced involved in modulating activation Nuclear Factor$x \mathrm{~B}(\mathrm{NF}-x \mathrm{~B})$. The activation of $\mathrm{NF}-x \mathrm{~B}$ has been demonstrated as a crucial role to regulate gene expressions in LPS-stimulated inflammatory responses, including $\mathrm{NO}$ and $\mathrm{PGE}_{2}(19)$.

Many plant extracts can be beneficial for ameliorating LPS-induced inflammation in folk medicine. For this purpose, many fruits as well as pomegranate have been widely used for their antiinflammatory properties (20). Many have reported the anti-inflammatory function of pomegranate in both in vitro and in vivo studies $(21,22)$. Pomegranate juice has been shown to promote antioxidant and antiinflammatory effects by inhibiting oxidative destruction of $\mathrm{NO}$. In vitro experiments have shown that pomegranate seed oil obtained by cold pressing suppresses cyclooxygenase and lipoxygenase enzymes (12). In another in vitro study, pomegranate extract has been shown to significantly suppress the subset of matrimal metalloproteinases of collagenase enzymes, which play a role in the construction and degradation of high resolution and extracellular matrix of the osteoarthritic joint (23). Treatment of pomegranate extract suppresses the degradation of proteoglycan stimulated by interleukin-1 in human femoral osteoarthritic chondrocytes and 
synthesis of cellular matrix metalloproteinases. In osteoarthritic chondrocyte cultures it has been shown that pomegranate extract inhibits collagen degradation, and may possibly suppress joint destruction in patients with osteoarthritis (24). However, the anti-inflammatory properties of pomegranate have not yet been demonstrated in clinical trials (12). Although these studies as well as other studies not mentioned here suggest that pomegranate extract hold good potential as antiinflammatory agent, data on the effects of punicalagin isolated from pomegranate husk is lacking $(23,24)$. The current study has been performed to enlighten the anti-inflammatory properties of punicalagin on LPS-induced RAW 264.7 macrophages.

Since the anti-inflammatory activity of punicalagin is independent of its cytotoxicity, we aimed primarily to determine the subcytotoxic concentrations of punicalagin in this study. For this reason, cell viability ratios were examined by MTT assay of RAW 264.7 macrophages incubated with different concentrations of punicalagin for 24 and 48 hours. The study showed that there was no significant change in viability of cells treated with $2.5,5,7.5$ and $10 \mu \mathrm{M}$ punicalagin, and punicalagin at concentrations of $20 \mu \mathrm{M}$ and higher caused significant cytotoxicity in RAW 264.7 macrophages. For this reason, in our study we used $0-10 \mu \mathrm{M}$ punicalagin concentrations having no cytotoxicity to RAW 264.7 macrophages.

Our study has shown that all applied concentration of punicalagin decreased LPSinduced NO production and gene expression of iNOS and COX-2 in RAW 264.7 macrophages, significantly. Similarly, all applied concentration of punicalagin decreased LPS-induced $\mathrm{PGE}_{2}$ production but only 7.5 and $10 \mu \mathrm{M}$ punicalagin decreased $\mathrm{PGE}_{2}$ production significantly. This indicates that punicalagin inhibited LPS-induced $\mathrm{NO}$ and $\mathrm{PGE}_{2}$ production and $i N O S$ and $C O X-2$ gene expression in RAW 264.7 macrophages in a dose-dependent manner and the decline in NO and $\mathrm{PGE}_{2}$ production by punicalagin is a result of the inhibition of iNOS and COX-2 gene expression.

In conclusion, it has been revealed that punicalagin isolated from Punica granatum inhibits inflammation via suppressing $i N O S$ and $C O X-2$ gene expression and $\mathrm{NO}$ and $\mathrm{PGE}_{2}$ production. The data shows that punicalagin holds great potential against the inflammatory pathway. This study has led to the conclusion that regular consumption of Punica granatum may help reducing the inflammatory response.

\section{References}

1. Tran HNK, Nguyen VT, Kim JA, et al. Antiinflammatory activities of compounds from twigs of Morus alba. Fitoterapia 2017; 120: 17-24.

2. Lee E, Kim SG, Park NY, et al. Antiinflammatory effects of KOTMIN13: A mixed herbal medicine in LPS-stimulated RAW 264.7 cells and mouse edema models. Pharmacogn Mag 2017; 13: 216-221.

3. Ghafoori H, Rezaei M, Mohammadi A. Antiinflammatory effects of novel thiazolidinone derivatives as bioactive heterocycles on RAW264.7 Cells. Iran J Allergy Asthma Immunol 2017; 16: 28-38.

4. Wang ZS, Chen LZ, Zhou HP, Liu XH, Chen FH. Diarylpentadienone derivatives (curcumin analogues): Synthesis and anti-inflammatory activity. Bioorg Med Chem Lett 2017; 27: 18031807.

5. Sanjeewa KK, Fernando IP, Kim EA, Ahn G, Jee Y, Jeon YJ. Anti-inflammatory activity of a sulfated polysaccharide isolated from an enzymatic digest of brown seaweed Sargassum horneri in RAW 264.7 cells. Nutr Res Pract 2017; 11: 3-10.

6. Liu F, Zhang $\mathrm{X}$, Ling $\mathrm{P}$, et al. Immunomodulatory effects of xanthan gum in LPS-stimulated RAW 264.7 macrophages. Carbohydr Polym 2017; 169: 65-74.

7. Panth N, Manandhar B, Paudel KR. Anticancer activity of Punica granatum (Pomegranate). Phytother Res 2017; 31: 568-578.

8. Kalaycıŏglu Z, Erim FB. Total phenolic contents, antioxidant activities, and bioactive ingredients of juices from pomegranate cultivars worldwide. Food Chem 2017; 221: 496-507.

9. Lyu A, Chen JJ, Wang HC, et al. Punicalagin protects bovine endometrial epithelial cells against lipopolysaccharide-induced inflammatory injury. J Zhejiang Univ Sci B 2017; 18: 481-491.

10. Sahebkar A, Simental-Mendía LE, Giorgini P, Ferri C, Grassi D. Lipid profile changes after pomegranate consumption: A systematic review and meta-analysis of randomized controlled trials. Phytomedicine 2016; 23: 1103-1112.

11. Kim YE, Hwang CJ, Lee HP, et al. Inhibitory effect of punicalagin on lipopolysaccharideinduced neuroinflammation, oxidative stress and memory impairment via inhibition of nuclear factor-kappaB. Neuropharmacology 2017; 117: 21-32.

12. Houston DM, Bugert J, Denyer SP, Heard CM. Anti-inflammatory activity of Punica granatum L. (Pomegranate) rind extracts applied topically to ex vivo skin. Eur J Pharm Biopharm 2017; 112: 30-37.

13. Gentile C, Allegra M, Angileri F, Pintaudi AM, Livrea MA, Tesoriere L. Polymeric 
proanthocyanidins from Sicilian pistachio (Pistacia vera L.) nut extract inhibit lipopolysaccharideinduced inflammatory response in RAW 264.7 cells. Eur J Nutr 2012; 51: 353-363.

14. Green LC, Glogowski J, Skipper PL, Wishnok JS, Tannenbaum SR. Analysis of nitrate, nitrite, and nitrate in biological fluids. Anal Biochem 1982; 126: 131-138.

15. An HJ, Kim IT, Park HJ, Kim HM, Choi JH, Lee KT. Tormentic acid, a triterpenoid saponin, isolated from Rosa rugosa, inhibited LPS-induced iNOS, COX-2, and TNF- $\alpha$ expression through inactivation of the nuclear factor- $x$ b pathway in RAW 264.7 macrophages. Int Immunopharmacol 2011; 11: 504-510.

16. Lopes PC. LPS and neuroinflammation: a matter of timing. Inflammopharmacology 2016; 24: 291 293.

17. Qin CC, Liu YN, Hu Y, Yang Y, Chen Z. Macrophage inflammatory protein-2 as mediator of inflammation in acute liver injury. World J Gastroenterol 2017; 23: 3043-3052.

18. Jackaman C, Tomay F, Duong L, et al. Aging and cancer: The role of macrophages and neutrophils. Ageing Res Rev 2017; 36: 105-116.
19. Kitanaka S. Chemical compounds in natural medicines that affect macropharges and adipocyte cells. Yakugaku Zasshi 2016; 136: 1195-1216.

20. Jungbauer A, Medjakovic S. Anti-inflammatory properties of culinary herbs and spices that ameliorate the effects of metabolic syndrome. Maturitas 2012; 71: 227-239.

21. Murakami A, Ohigashi H. Targeting NOX, iNOS and COX-2 in inflammatory cells: chemoprevention using food phytochemicals. Int J Cancer 2007; 121: 2357-2363.

22. McDaniel ML, Kwon G, Hill JR, Marshall CA, Corbett JA. Cytokines and nitric oxide in islet inflammation and diabetes. Proc Soc Exp Biol Med 1996; 211: 24-32.

23. Ghoochani N, Karandish M, Mowla K, Haghighizadeh MH, Jalali MT. The effect of pomegranate juice on clinical signs, matrix metalloproteinases and antioxidant status in patients with knee osteoarthritis. J Sci Food Agric 2016; 96: 4377-4381.

24. Jean-Gilles D, Li L, Vaidyanathan VG, et al. Inhibitory effects of polyphenol punicalagin on type-II collagen degradation in vitro and inflammation in vivo. Chem Biol Interact 2013; 205: 90-99. 